\title{
COVID-19: Cardiovascular involvement without pulmonary signs.
}

A Tito Paladino $\mathrm{F}^{1 *}$, Bruno Valdigem ${ }^{2}$, Hugo Lopes $^{3}$, Roberto Tuma ${ }^{2}$, Bruno Normande ${ }^{2}$, Natalia Massoni $^{1}$, Natasha dos Santos ${ }^{2}$, Andrea Vilela ${ }^{1}$, Ibraim Francisco ${ }^{2}$, Jorge Assef ${ }^{1}$

${ }^{1}$ Department of Echocardiography, Instituto Dante Pazzanese de Cardiologia, São Paulo-SP, Brazil

${ }^{2}$ Department of Cardiovascular Imaging, Instituto Dante Pazzanese de Cardiologia, São Paulo-SP, Brazil

${ }^{3}$ Department of Electrophysiology, Instituto Dante Pazzanese de Cardiologia, São Paulo-SP, Brazil

\section{Abstract}

Sars-COV2 usually has a flu-like condition with possible progression to lung involvement. Isolated cardiac involvement has a slight frequency and present as arrhythmias, pericarditis or myocarditis. This is a rare case of COVID-1 patient, without pulmonary involvement with pericarditis, pericardial effusion and focal left ventricular impaired contraction.

Keywords: Echocardiography, Cardiac magnetic resonance, Computed tomography, Pericardial effusion.

Accepted on August 04, 2020

\section{Case Presentation}

Male, physician, $40 \mathrm{y}$ presenting nasal congestion without a runny nose for 3 days. Discreet degree headache and odynophagia. Due to the history of attending hospitals with patients diagnosed with COVID-19, he performed a swab on April 19, being positive. He performed a chest tomography on the same day without any finding suggestive of pulmonary involvement, but with a slight more evident pericardial effusion adjacent to the right ventricle free wall (Figure 1).
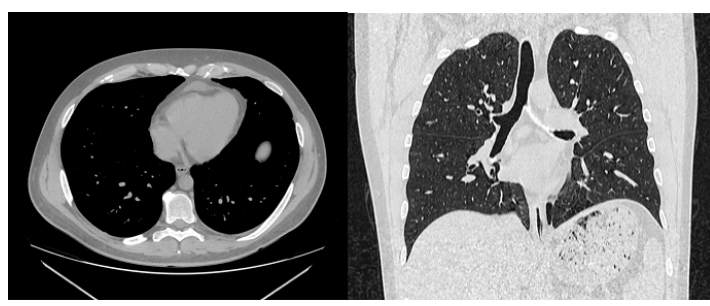

Figure 1. (A) CT-Midly pericardial effusion (white arrow) (B) No pulmonary involvement.

Echocardiogram (20/04) preserved ejection fraction ( $\mathrm{LVEF}=66 \%$, Simpson), with reduced global longitudinal strain ( $\mathrm{SLG}=-16)$, most evident in anterior, anteroseptal and anterolateral wall (basal segments) (Figure 2).

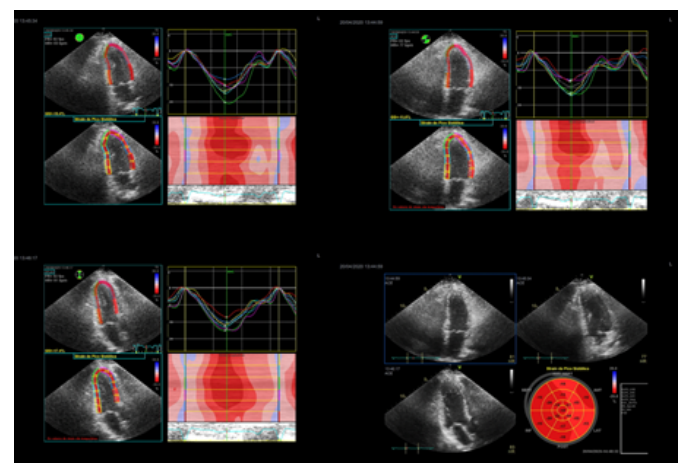

Figure 2. Strain: Deficit in basal segments (anterosseptal, anterior and anterolateral walls).
Cardiac Magnetic Resonance (20/04) pericardial effusion adjacent to the free wall of the right ventricle, with slight associated late enhancement, including adjacent to lateral wall. The septum had an almost undetectable contractile deficit, with other segments preserved (Figure 3).

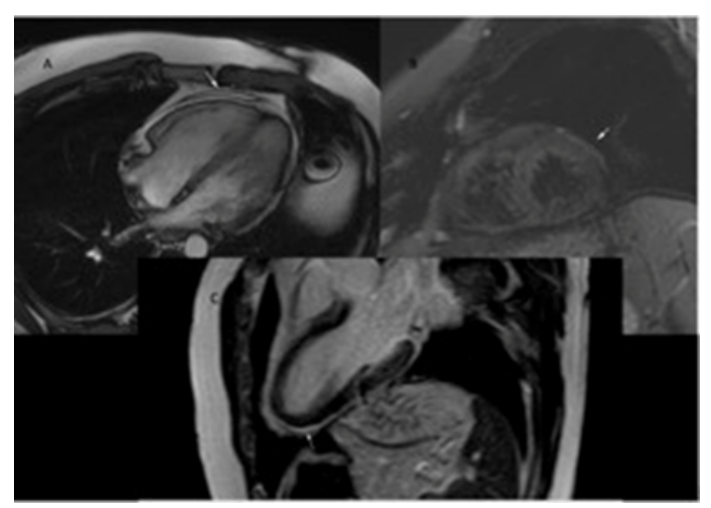

Figure 3. (A) CMR Cine (4 chambers)-Midly pericardial effusion (B) $C M R$ (triple)-Edema at "lateral" pericardium (C) CMR (Delayed Gadolinium Enhancement)-Pericarditis.

Eletrocardiogram (20/04) nothing but sinus rhythm.

CRP and Troponin (20/04) Normal values

\section{Resume}

Acute respiratory infections, including influenza, respiratory syncytial virus, and bacterial pneumonias, are well-recognized triggers for cardiovascular diseases (CVD), and the underlying CVD can lead to a worse prognosis for such infections. 
Both SARS and middle east respiratory syndrome, viruses with similarities to COVID-19, have shown deleterious effects on the cardiovascular system. We can mention: arrhythmias (bradycardias and tachycardias), changes in diastolic function, transient cardiomegaly, hypotension, myocarditis, pericardial effusion and acute coronary syndrome.

The SARS-CoV-2 (COVID-19) infection causes a severe respiratory illness with many epidemiologic, clinical, radiologic, and laboratory findings. The 3 most common symptoms of COVID-19 are fever, cough, and shortness of breath, but we can have muscle pain, anorexia, malaise, sore throat, nasal congestion, dyspnea, and headache [1].

COVID-19 apparently affects the myocardium causing myocarditis. It is reasonable to expect that advanced and critical cases have more severe effects on the cardiovascular system due to more intense inflammatory response. Some severe myocarditis with reduced systolic function has been reported after COVID-19, and is an important prognostic factor.

Cardiac biomarker studies suggest a high prevalence of cardiac injury in hospitalized patients. Presence of any grade of cardiac injury, myocarditis, and ARDS are strong and independent factors associated with high mortality. Pericarditis is not described in the literature till now, but we have cases with pericardial effusion leading to tamponade [2].

\section{Differential Diagnosis}

Both SARS and Middle East respiratory syndrome, viruses with characteristics similar to COVID-19, have shown deleterious effects on the cardiovascular system. We can mention: arrhythmias (bradycardias and tachycardias), changes in diastolic function, transient cardiomegaly, hypotension, myocarditis, pericardial effusion and acute coronary syndrome.

The SARS-CoV-2 (COVID-19) infection causes a severe respiratory illness with many epidemiologic, clinical, radiologic, and laboratory findings. Transmission of SARSCoV-2 seems to be primarily from person to person via close contact, through respiratory droplets, with a mean incubation period of 5.2 days.

The 3 most commom symptoms of COVID-19 are fever, cough, and shortness of breath, but we can have muscle pain, anorexia, malaise, sore throat, nasal congestion, dyspnea, and headache.

COVID-19 Apparently affect the myocardium causing myocarditis. It is reasonable to expect that severe and critical cases have more severe effects on the cardiovascular system due to more intense inflammatory response. Some severe myocarditis with reduced systolic function have been reported after COVID-19, and is an important prognostic factor.
Cardiac biomarker studies suggest a high prevalence of cardiac injury in hospitalized patients. Presence of any grade of cardiac injury, myocarditis, and ARDS are strong and independent factors associated with high mortality. Pericarditis is not as described in the literature, but we have cases with pericardial effusion leading to tamponade [3].

\section{Management}

Symptomatic medications and rest.

\section{Discussion}

Early onset of symptoms, and rapid and effective medical care, we may have highlight early cardiovascular changes to the point that there was no time to increase CRP or troponin. A small extension of the pericardium was affected, as well as only a small volume of pericardial effusion was detected. This demonstrates that the disease brings systemic changes with an inflammatory origin, and may start with a poor cardiological condition even without pulmonary involvement in the tomographic study. So, we conclude that a detailed analysis of all "systems" must be performed since it is a "new" disease without a well-established course. Many patients may have only cardiovascular disease, and with the routine tests currently used, they would go unnoticed. We need more research and investment to detail the natural history of this new disease.

Among the complementary exams, computed tomography of the chest is indicated to assist in the diagnosis definition, always in correlation with clinical and laboratory data. CT scans has sensitivity between $61-97 \%$, with focal ground-glass opacities or consolidations as findings, with bilateral, peripheral and basal involvement. However, could be normal in about $50 \%$ of cases, in the initial phase of the disease ( 0 to 2 days of symptoms) [4].

Cardiac magnetic resonance was performed to investigate the suspicion of active pericarditis as the cause of her chest pain, which demonstrated significantly increased pericardial signal on short-tau inversion recovery (STIR) imaging, consistent with pericardial oedema. On delayed-enhancement imaging, there was significantly increased pericardial signal, consistent with active pericardial inflammation. These findings are consistent with active pericarditis with pericardial inflammation/edema [5].

\section{Conclusion}

The pathogenesis of cardiac involvement associated with SARS-CoV-2 may reflect a process of replication and dissemination of the virus through the blood or the lymphatic system from the respiratory tract. However, to our knowledge, there are no reports of influenza virus or coronavirus RNA in the heart, to date. Alternatively, SARS-CoV-2 could trigger an exaggerated inflammatory response that can cause cardiac injury, and this could justify the use of corticosteroids to attenuate inflammation. Evidence of a significant inflammatory cell infiltration has been reported in the alveoli of patients with acute respiratory distress syndrome associated with SARS- 
CoV-2 infection, 10 and this could explain the use of corticosteroids in patients with COVID-19 (up to 58\% in a series of critically ill patients13).

\section{Learning Objectives}

A case of a patient with cardiovascular covid affection before lung alterations in $\mathrm{CT}$.

1. To be able to diagnose cardiac affection by Covid.

2. To be able to better screen Covid full body infecton.

\section{Funding}

No external funding was utilized to support this study.

\section{Disclosures}

The authors have no financial relationships to disclose.

\section{References}

1. Hua A, O'Gallagher K, Sado D, et al. Life-threatening cardiac tamponade complicating myo-pericarditis in COVID-19. Eur Heart J. 2020;41(22):2130.

2. Ariyarajah V, Jassal DS, Kirkpatrick I, et al. The utility of cardiovascular magnetic resonance in constrictive pericardial disease. Cardiol Rev. 2009;17(2): 77-82.
3. Madjid M, Safavi-Naeini P, Solomon SD, et al. Potential Effects of Coronaviruses on the Cardiovascular System: A Review. JAMA Cardiol. 2020.

4. D. Hope M, A. Raptis C, S. Henry T.Chest Computed Tomography for Detection of Coronavirus Disease 2019 (COVID-19): Don't Rush the Science. Ann Intern Med. 2020: M20-1382.

5. Bogaert J, Francone M. Pericardial disease: value of CT and MR imaging. Radiology.2013;267(2):340-56.

\section{*Correspondence to}

A Tito Paladino F

Department of Echocardiography

Instituto Dante Pazzanese de Cardiologia

Avenida Dante Pazzanese 500

Dr. Dante Pazzaneseavenue

São Paulo-SP

Brazil

E-mail: atpf40@gmail.com 